\title{
SMOOTH BOUNDED STRICTLY AND WEAKLY PSEUDOCONVEX DOMAINS CANNOT BE BIHOLOMORPHIC
}

\author{
BY STEVEN BELL
}

There is no Riemann mapping theorem in the theory of functions of several complex variables; nor is there a Riemann nonmapping theorem. In fact, until recently, it was not known whether it is possible for a smooth bounded strictly pseudoconvex domain to be biholomorphically equivalent to a smooth bounded weakly pseudoconvex domain. The paper [1] answers this question in the negative by proving

THEOREM 1. If $D_{2}$ is a smooth bounded pseudoconvex domain, and $D_{1}$ is a smooth bounded domain whose $\bar{\partial}$-Neumann problem satisfies global regularity estimates, then biholomorphic mappings between $D_{1}$ and $D_{2}$ extend smoothly to the boundary.

In particular, it is known (Kohn [6]) that the $\bar{\partial}$-Neumann problem satisfies global regularity estimates in smooth bounded strictly pseudoconvex domains. Hence, a biholomorphic mapping between a smooth bounded strictly pseudoconvex domain and a smooth bounded weakly pseudoconvex domain would extend smoothly to the boundary. Since strict pseudoconvexity is preserved under biholomorphic mappings which extend to be $C^{2}$ up to the boundary, both domains must be strictly pseudoconvex.

Other domains for which the $\bar{\partial}$-Neumann problem is known to satisfy global regularity estimates include smooth bounded weakly pseudoconvex domains with real analytic boundaries [7], [2] and certain domains of finite type [7].

The proof of Theorem 1 exploits the transformation rule for the Bergman projection. If $P_{i}$ denotes the Bergman orthogonal projection of $L^{2}\left(D_{i}\right)$ onto its subspace of holomorphic functions, $i=1,2$, and if $f: D_{1} \rightarrow D_{2}$ is a biholomorphic mapping, then

$$
P_{1}(u \cdot(\phi \circ f))=u \cdot\left(\left(P_{2} \phi\right) \circ f\right)
$$

where $u=\operatorname{Det}\left[f^{\prime}\right]$ and $\phi \in L^{2}\left(D_{2}\right)$. It is possible to construct functions $\phi$ which vanish to arbitrarily high order on $b D_{2}$ such that $P_{2} \phi \equiv 1$. If $\phi$ is such a function which vanishes to a high enough order on $b D_{2}$ to make $u \cdot(\phi \circ f)$ smooth up to the boundary, then $u$ is the projection of a function which is smooth up to the

Received by the editors August 1, 1980.

1980 Mathematics Subject Classification. Primary 32H99; Secondary 35N15, 32A40.

(c) 1981 American Mathematical Society $0002-9904 / 81 / 0000-0011 / \$ 01.50$ 
boundary. Since global estimates for the $\bar{\partial}$-problem in $D_{1}$ imply global estimates for $P_{1}$, we conclude that $u$ is smooth up to the boundary.

A similar argument reveals that $u \cdot(g \circ f)$ is smooth up to the boundary whenever $g$ is a holomorphic function on $D_{2}$ which is smooth up to the boundary.

The final steps in the proof of Theorem 1 depend on Kohn's theory of the $\bar{\partial}$-Neumann problem with weight functions [8] and a special Sobolev inequality for holomorphic functions: If $g$ and $h$ are holomorphic functions on a smooth bounded domain $D$, then

$$
\left|\int_{D} h \bar{g}\right| \leqslant C\|h\|_{s}\|g\|_{-s}
$$

where $\|h\|_{s}^{2}=\Sigma_{|\alpha| \leqslant s}\left\|D^{\alpha} h\right\|_{L^{2}(D)}^{2}$ is the usual Sobolev s-norm and

$$
\|g\|_{-s}=\operatorname{Sup}_{\substack{\phi \in C_{0}^{\infty}(D) \\\|\phi\|_{s}=1}}\left|\langle g, \phi\rangle_{L^{2}(D)}\right| .
$$

The constant $C$ only depends on $D$ and the positive integer $s$. This inequality is unique to holomorphic functions and must not be confused with the standard generalized Schwarz inequality.

Theorem 1 generalizes and improves several earlier results on biholomorphic mappings due to Henkin [5], Pinčuk [9], Fefferman [4], Diederich and Fornaes: [3]. The Henkin, Pinčuk, Diederich and Fornaess results also apply to proper holomorphic mappings. At the present time, the techniques used in the proof of Theorem 1 do not seem to adapt to the proper mapping case.

\section{REFERENCES}

1. S. Bell, Biholomorphic mappings and the $\bar{\partial}$-problem, Ann. of Math. (2) (to appear).

2. K. Diederich and J. E. Fornaess, Pseudoconvex domains with real-analytic boundary, Ann. of Math. (2) 107 (1978), 371-384.

3. - Proper holomorphic maps onto pseudoconvex domains with real analytic boundary, Ann. of Math. (2) 110 (1979), 575-592.

4. C. Fefferman, The Bergman kernel and biholomorphic mappings of pseudoconvex domains, Invent. Math. 26 (1974), 1-65.

5. G. Henkin, An analytic polyhedron is not holomorphically equivalent to a strictly pseudoconvex domain, Soviet Math. Dokl. 14 (1973), 858-862.

6. J. J. Kohn, Harmonic integrals on strongly pseudoconvex manifolds. I, II, Ann of Math. (2) 78 (1963), 112-148; 79 (1964), 450-472.

7. - Subellipticity of the $\bar{\partial}$-Neumann problem on pseudoconvex domains: sufficient conditions, Acta Math. 142 (1979), 79-122.

8. - Global regularity for $\bar{\partial}$ on weakly pseudoconvex manifolds, Trans. Amer. Math. Soc. 181 (1973), 273-292.

9. S. Pinčuk, On proper holomorphic mappings of strictly pseudoconvex domains, Siberian Math. J. 15 (1974), 644-649.

DEPARTMENT OF MATHEMATICS, PRINCETON UNIVERSITY, PRINCETON, NEW JERSEY 08540 